\title{
Analysis of a Circular Waveguide Loaded with Thick Annular Metal Discs for Wide-Band Gyro-TWTs
}

\author{
Vishal Kesari, P. K. Jain, and B. N. Basu
}

\begin{abstract}
The cold (beam-absent) analysis of an all-metal structure consisting of a circular waveguide loaded with axially periodic annular discs was developed in the fast-wave regime for potential application in wide-band gyro-traveling-wave tubes (TWTs) in the millimeter-wave frequency range. The analysis includes the effect of higher order standing-wave modes in the disc-occupied region, as well as higher order space harmonic propagating modes in the disc-free region of the structure. The analysis also takes into account the effect of the finite disc thickness. The dispersion characteristics of the structure obtained by the analysis have been validated against those reported elsewhere using an alternative coupled-integral-equation technique and also against those obtained using commercial simulation code HFSS. The dependence of the eigenvalue and dispersion characteristics of the structure on the disc parameters, namely, the disc hole radius, periodicity, and thickness was studied. The optimum disc parameters corresponding to the widest frequency band over which the dispersion curve can be straightened were suggested for widening the bandwidth of coalescence between the waveguide-mode and beam-mode dispersion characteristics for wide-band gyro-TWT performance. The optimum disc parameters for wide device bandwidths and high device gains were also predicted with the help of the small-signal gain equation of a gyro-TWT.
\end{abstract}

Index Terms-Disc-loaded waveguide, gyrotron, millimeterwave amplifier, periodic electromagnetic structure, wide-band gyro-traveling-wave tube (gyro-TWT).

\section{INTRODUCTION}

$\mathbf{T}$ HE DEVELOPMENT of fast-wave gyro-amplifiers like gyro-klystrons and gyro-traveling-wave tubes (gyroTWTs), which overcomes the high-power and high-frequency limitations of conventional klystrons and TWTs in the family of microwave tubes, opens up applications in high power, millimeter-wave radars of high resolution, and communication systems of high information density [1]. Clearly, the conventional slow-wave devices like TWTs, due to reduced sizes of their interaction structures, cannot compete with the fast-wave devices like gyro-TWTs in the high-power and high-frequency (millimeter-wave) regime. Consequently, this has aroused considerable interest in the development of gyro-devices like gyro-TWTs, despite the fact that in bremsstrahlung devices like gyro-devices, it is required to generate an electron beam of gyrating electrons using a magnetron injection gun for the

Manuscript received October 12, 2004; revised March 22, 2005. The work of V. Kesari was supported by the Centre of Advanced Studies (UGC).

The authors are with the Centre of Research in Microwave Tubes, Department of Electronics Engineering, Institute of Technology, Banaras Hindu University, Varanasi-221 005, India (e-mail: vishal_kesari@rediffmail.com; bnbasu@bhu.ac.in).

Digital Object Identifier 10.1109/TPS.2005.852393 small-orbit configuration and a cusp gun for the large-orbit configuration, unlike in conventional O-type microwave tubes like TWTs requiring a linear electron beam that can be formed by a simple Pierce gun [1], [2].

A gyro-TWT has a wider bandwidth potential than a gyro-klystron, since the former does not use interaction cavities unlike the latter. In a gyro-TWT, one may taper the cross section of the waveguide interaction structure and simultaneously profile the magnetic field to broad-band the device; a method that, however, reduces the device gain [3] and [4]. Alternatively, one may load the waveguide wall by a dielectric lining to straighten the dispersion characteristics over a wider frequency range for wide-band coalescence between the beam-mode and waveguide-mode dispersion characteristics and, consequently, for wide device bandwidths [5]. The method, however, entails the risk of dielectric charging and heating [5]. This calls for an all metal loading of the waveguide for controlling the dispersion characteristics [4], [6]. One such structure is a circular waveguide loaded with axially periodic metal discs.

The analysis of a disc-loaded circular waveguide was carried out in the past both for linear accelerators in the slowwave regime, for instance, in [7] and [8], and for gyro-TWTs in the fast-wave regime, for instance, in [6] and [9]. The surface impedance model for the analysis of a disc-loaded circular waveguide, in which the interface between the disc-occupied and disc-free regions is treated as a homogeneous reactive surface, was used for closely spaced discs [10]. Dybdal et al. [11] applied the impedance boundary approach to study a corrugated rectangular waveguide. Amari et al. [12], [13] analyzed the structure using the coupled-integral-equation technique and found the propagation constants of Floquet's modes from the classical eigenvalues of a characteristic matrix. Esteban and Rebollar [14] used the modal expansion technique considering the disc-loaded waveguide of finite disc thickness to be constituted by consecutive smooth-wall waveguides, such that every alternate waveguide has the wall radius equal to either the overall waveguide radius or the disc hole radius, and, hence, studied the hybrid modes and the complex solution of propagation constants for smaller disc hole radii. Scattering matrix formalism for a rectangular corrugated waveguide due to Wagner et al. [15] could take into account the precise shape of the corrugation

Choe and Uhm [9] analyzed a disc-loaded circular waveguide in the fast-wave regime for application in gyro-TWTs, including the effects of the space harmonics generated due to the axial periodicity of the structure. They considered the lowest order standing-wave mode in the disc-occupied region and the fundamental propagating-wave mode in the disc-free region. Kesari 


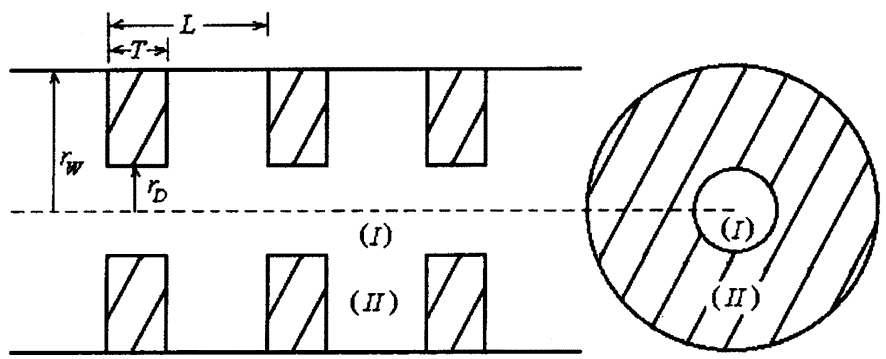

Fig. 1. Schematic of a circular waveguide loaded with thick metal discs of uniform periodicity showing the disc parameters and the two structure regions (I and II).

et al. [6] extended the analysis of Choe and Uhm [9] to consider higher order modes in both the disc-free and disc-occupied regions. However, for the sake of simplicity, infinitesimally thin discs were assumed in the analyzes of both Choe and Uhm [9] and Kesari et al. [6]. In this paper, the fast-wave analysis of a disc-loaded circular waveguide is generalized considering the effect of higher order modes, in both the disc-occupied and disc-free regions, and that of finite disc thickness (Section II). Moreover, how the effect of the disc parameters on the control of the dispersion characteristics of the structure predicted by the present analysis would influence the gain-frequency response of a gyro-TWT has also been studied with the help of a simple small-signal gain equation of the device (Section III).

\section{ANALYSIS}

The structure analyzed in the fast-wave regime consists of a circular waveguide loaded with thick annular, axially periodic metal discs (Fig. 1). The method of analysis closely follows the one previously given for infinitesimally thin discs reported in Kesari et al. [6]. The method is, however, improved in this paper by including the effect of the finite thickness of discs. Moreover, in the present analysis, the propagation constant obtained by the cold (beam-absent) analysis (Section II-A) is fed into the small-signal gain equation of a gyro-TWT, the latter interpretable from the beam-present dispersion relation of a gyro-TWT (Section II-B), [2], [4], [5].

The structure is divided into the disc-free region $0 \leq r<$ $r_{D}, 0<z<\infty$, labeled as region I, and the disc-occupied region $r_{D} \leq r<r_{W}, 0<z<(L-T)$, labeled as region II, where $r_{D}$ is the disc hole radius, $r_{W}$ the waveguide wall radius, $L$ the disc periodicity, and $T$ the disc thickness (Fig. 1). The analysis is carried out for TE modes $\left(E_{z}=0\right)$ keeping in mind the potential application of the structure in a gyro-TWT which operates at or near the grazing intersection or coalescence between the beam-mode and waveguide-mode dispersion characteristics where the growth rate of TE modes is much more significant than that of TM modes [16]. Further, considering nonazimuthally varying modes $(\partial / \partial \theta=0)$, the relevant field expressions for regions I and II may be written, in the cylindrical system of coordinates $(r, \theta, z)$ as [6]

$$
\begin{aligned}
& H_{z}^{\mathrm{I}}=\sum_{n=-\infty}^{+\infty} A_{n}^{\mathrm{I}} J_{0}\left\{\gamma_{n}^{\mathrm{I}} r\right\} \exp j\left(\omega t-\beta_{n}^{\mathrm{I}} z\right) \\
& E_{\theta}^{\mathrm{I}}=j \omega \mu_{0} \sum_{n=-\infty}^{+\infty} \frac{1}{\gamma_{n}^{\mathrm{I}}} A_{n}^{\mathrm{I}} J_{0}^{\prime}\left\{\gamma_{n}^{\mathrm{I}} r\right\} \exp j\left(\omega t-\beta_{n}^{\mathrm{I}} z\right)
\end{aligned}
$$

and

$$
\begin{aligned}
& H_{z}^{\mathrm{II}}=\sum_{m=1}^{\infty} A_{m}^{\mathrm{II}} Z_{0}\left\{\gamma_{m}^{\mathrm{II}} r\right\} \exp (j \omega t) \sin \left(\beta_{m}^{\mathrm{II}} z\right) \\
& E_{\theta}^{\mathrm{II}}=j \omega \mu_{0} \sum_{m=1}^{\infty} \frac{1}{\gamma_{m}^{\mathrm{II}}} A_{m}^{\mathrm{II}} Z_{0}^{\prime}\left\{\gamma_{m}^{\mathrm{II}} r\right\} \exp (j \omega t) \sin \left(\beta_{m}^{\mathrm{II}} z\right)
\end{aligned}
$$

where (see (5) at the bottom of the page.) The superscripts I and II refer to the disc-free region I and the disc-occupied region II, respectively (Fig. 1). The subscript $n$ represents the spaceharmonic number referring to region I supporting space harmonics generated due to the axial periodicity of the structure, and the subscript $m$ represents the modal harmonic number referring to region II supporting standing waves caused by reflection at the metal discs [6], [9]. $A_{n}^{\mathrm{I}}$ and $A_{m}^{\mathrm{II}}$ are the field constants. $J_{0}$ and $Y_{0}$ are the zeroth order Bessel functions of the first and second kinds, respectively, the primes with the Bessel functions indicating the derivatives with respect to respective arguments. $\gamma_{n}^{\mathrm{I}}\left(=\left[k^{2}-\left(\beta_{n}^{\mathrm{I}}\right)^{2}\right]^{1 / 2}\right)$ and $\gamma_{m}^{\mathrm{II}}\left(=\left[k^{2}-\left(\beta_{m}^{\mathrm{II}}\right)^{2}\right]^{1 / 2}\right)$ are the radial propagation constants, $k$ being the free-space propagation constant. $\beta_{n}^{\mathrm{I}}\left(=\beta_{0}^{\mathrm{I}}+2 \pi n / L\right)(n=0, \pm 1, \pm 2, \ldots \pm \infty)$ is the axial phase propagation constant referring to region I that obeys Floquet's theorem [8] as applied to the present structure that coincides with itself as it is translated through an axial distance equal to the periodicity of the structure $L$ (Fig. 1). $\beta_{m}^{\mathrm{II}}=m \pi /(L-T)$ $(m=1,2,3, \ldots, \infty)$ is the axial phase propagation constant referring to region II, which is the groove region of the axial length $L-T$ supporting integral multiple of half wavelengths [6].

\section{A. Cold (Beam-Absent) Dispersion Relation}

The cold (beam-absent) dispersion relation of the disc-loaded circular waveguide may be obtained with the help of the field expressions (1)-(4) and the following boundary conditions:

$$
\text { at } \begin{aligned}
r=r_{D}: & \\
H_{z}^{\mathrm{I}} & =H_{z}^{\mathrm{II}} \quad 0<z<(L-T) \\
E_{\theta}^{\mathrm{I}} & = \begin{cases}E_{\theta}^{\mathrm{II}}, & 0<z<(L-T) \\
0, & (L-T)<z<L .\end{cases}
\end{aligned}
$$

The boundary condition (6) states that the axial (tangential) components of magnetic field intensity is continuous at the interface between the regions I and II $(0<z<(L-T))$. The boundary condition (7) states that 1 ) the azimuthal (tangential)

$$
\left.\begin{array}{l}
Z_{0}\left\{\gamma_{m}^{\mathrm{II}} r\right\}=\left(Y_{0}^{\prime}\left\{\gamma_{m}^{\mathrm{II}} r_{W}\right\} J_{0}\left\{\gamma_{m}^{\mathrm{II}} r\right\}-J_{0}^{\prime}\left\{\gamma_{m}^{\mathrm{II}} r_{W}\right\} Y_{0}\left\{\gamma_{m}^{\mathrm{II}} r\right\}\right) / Y_{0}^{\prime}\left\{\gamma_{m}^{\mathrm{II}} r_{W}\right\} \\
Z_{0}^{\prime}\left\{\gamma_{m}^{\mathrm{II}} r\right\}=\left(Y_{0}^{\prime}\left\{\gamma_{m}^{\mathrm{II}} r_{W}\right\} J_{0}^{\prime}\left\{\gamma_{m}^{\mathrm{II}} r\right\}-J_{0}^{\prime}\left\{\gamma_{m}^{\mathrm{II}} r_{W}\right\} Y_{0}^{\prime}\left\{\gamma_{m}^{\mathrm{II}} r\right\}\right) / Y_{0}^{\prime}\left\{\gamma_{m}^{\mathrm{II}} r_{W}\right\}
\end{array}\right\} .
$$


components of electric field intensity is continuous at the interface between the regions I and II $(0<z<(L-T))$ and that 2 ) the azimuthal (tangential) component of electric field intensity is zero at the inner edge of thickness $T$ of the metal disc $((L-T)<z<L)$.

One may express the field constants $(m=1,2,3, \ldots)$, in terms of a series involving $A_{n}^{\mathrm{I}}(-\infty<n<\infty)$. For this purpose, one may substitute the field expressions (1) and (3) into the boundary condition (6), multiply it by $\sin \left(\beta_{m}^{\mathrm{II}} z\right)$, then integrate between $z=0$ and $L-T$, and make use of the orthogonal properties of trigonometric functions. Thus, one obtains

$$
A_{m}^{\mathrm{II}}=\sum_{n=-\infty}^{\infty} A_{n}^{\mathrm{I}} P_{n m}
$$

where

$$
P_{n m}=\frac{2}{L} \frac{J_{0}\left\{\gamma_{n}^{\mathrm{I}} r_{D}\right\}}{Z_{0}\left\{\gamma_{m}^{\mathrm{II}} r_{D}\right\}} \frac{\beta_{m}^{\mathrm{II}}\left[1-(-1)^{m} \exp \left(-j \beta_{0}^{\mathrm{I}} L\right)\right]}{\left(\beta_{m}^{\mathrm{II}}\right)^{2}-\left(\beta_{n}^{\mathrm{I}}\right)^{2}} .
$$

Similarly, one may obtain another series expression, similar to $(8)$, for $A_{m}^{\mathrm{II}}(m=1,2,3, \ldots)$, but now with the help of the field expressions (2) and (4) [instead of (1) and (3)] and the boundary condition (7) [instead of (6)], and by changing the integration limits to $z=0$ and $L$ (instead of $z=0$ and $L-T$ ), as follows:

$$
A_{m}^{\mathrm{II}}=\sum_{n=-\infty}^{\infty} A_{n}^{\mathrm{I}} Q_{n m}
$$

where

$$
\begin{aligned}
Q_{n m} & =\frac{2}{L} \frac{\gamma_{m}^{\mathrm{II}}}{\gamma_{n}^{\mathrm{I}}} \frac{J_{0}^{\prime}\left\{\gamma_{n}^{\mathrm{I}} r_{D}\right\}}{Z_{0}^{\prime}\left\{\gamma_{m}^{\mathrm{II}} r_{D}\right\}} \\
& \times \frac{\beta_{m}^{\mathrm{II}}-\left(\beta_{m}^{\mathrm{II}} \cos \left(\beta_{m}^{\mathrm{II}} L\right)+j \beta_{n}^{\mathrm{I}} \sin \left(\beta_{m}^{\mathrm{II}} L\right)\right) \exp \left(-j \beta_{0}^{\mathrm{I}} L\right)}{\left(\beta_{m}^{\mathrm{II}}\right)^{2}-\left(\beta_{n}^{\mathrm{I}}\right)^{2}} .
\end{aligned}
$$

The following relation results by equating the right hand sides of (8) and (9):

$$
\sum_{n=-\infty}^{\infty} A_{n}^{\mathrm{I}}\left(P_{n m}-Q_{n m}\right)=0
$$

Choosing to take the same number $q$, say, of the values of the standing wave modal number $(m)$ as that of space harmonic number $(n)$ (typically, corresponding to $q=7, n=0, \pm 1, \pm 2, \pm 3$, and $m=1,2,3,4,5,6,7)$, one can form $q$ number of simultaneous equations in the field constants $A_{n}^{\mathrm{I}}$ (typically, seven equations in $A_{0}^{\mathrm{I}}, A_{ \pm 1}^{\mathrm{I}} A_{ \pm 2}^{\mathrm{I}}, A_{ \pm 3}^{\mathrm{I}}$ ) with the help of (10). The condition for nontrivial solution that the determinant formed by the coefficients of these constants in these equations is zero yields the following dispersion relation of the structure:

$$
\begin{aligned}
& \operatorname{det} \mid M_{n m} J_{0}\left\{\gamma_{n}^{\mathrm{I}} r_{D}\right\} Z_{0}^{\prime}\left\{\gamma_{m}^{\mathrm{II}} r_{D}\right\} \\
& -Z_{0}\left\{\gamma_{m}^{\mathrm{II}} r_{D}\right\} J_{0}^{\prime}\left\{\gamma_{n}^{\mathrm{I}} r_{D}\right\} \mid=0 \\
& \quad(-\infty<n<\infty, 1 \leq m<\infty)
\end{aligned}
$$

where

$$
\begin{aligned}
& M_{n m}= \\
& \frac{\gamma_{n}^{\mathrm{I}} \beta_{m}^{\mathrm{II}}\left[1-(-1)^{m} \exp \left(-j \beta_{0}^{\mathrm{I}} L\right)\right]}{\gamma_{m}^{\mathrm{II}}\left[\beta_{m}^{\mathrm{II}}-\left(\beta_{m}^{\mathrm{II}} \cos \left(\beta_{m}^{\mathrm{II}} L\right)+j \beta_{n}^{\mathrm{I}} \sin \left(\beta_{m}^{\mathrm{II}} L\right)\right) \exp \left(-j \beta_{0}^{\mathrm{I}} L\right)\right]} .
\end{aligned}
$$

\section{B. Small-Signal Gain Equation}

One may interpret the dispersion relation (beam-present) of a gyro-TWT to write, in a form similar to that obtained by Pierce [17] for the gain in decibels of a conventional TWT, the following expression for $G$, the gain in decibels, of a gyro-TWT [2], [4], [5]:

$$
G=A+B C N
$$

where

$$
\left.\begin{array}{l}
A=-20 \log _{10}\left|\left(1-\delta_{2} / \delta_{1}\right)\left(1-\delta_{3} / \delta_{1}\right)\right| \\
B=40 \pi\left(\log _{10} e\right) x_{1} \cong 54.6 x_{1} \\
C=\left(K I_{0} / 4 V_{0}\right)^{1 / 3} \\
N=\beta_{0}^{\mathrm{I}} l / 2 \pi
\end{array}\right\} .
$$

In (13), $\delta_{1}, \delta_{2}$, and $\delta_{3}$, occurring in the expressions for $A$, known as the launching loss [2], [4], [5] are the roots of the cubic equation $\delta(\delta+j b)^{2}=j$, where $b\left(=\left(\omega-\beta_{0}^{\mathrm{I}} v_{z}-s \omega_{c} / \gamma\right) / \beta_{0}^{\mathrm{I}} v_{z} C\right)$ is a measure of the deviation from the resonance of the Doppler-shifted frequency from the harmonic of the relativistic electron cyclotron frequency. $\omega_{c}$ is the nonrelativistic electron cyclotron angular frequency. $s$ is the beam-harmonic mode number. $v_{z}\left(=\left[\left(\gamma^{2}-1\right) /\left(1+\alpha_{0}^{2}\right)\right]^{1 / 2} / \gamma\right)$ is the axial velocity of electrons, where $\alpha_{0}\left(=v_{t} / v_{z}\right)$ is the beam pitch factor and $\gamma\left(=\left[1-\left(v_{z}^{2}+v_{t}^{2}\right) / c^{2}\right]^{-1 / 2}\right)$ is the relativistic mass factor, which may be found in terms of the beam voltage $V_{0}$ by equating the relativistic kinetic energy $\left(=(\gamma-1) m_{e 0} c^{2}\right)$ with the potential energy $\left(=|e| V_{0}\right)$ of the beam as $\gamma=1+|e| V_{0} / m_{e 0} c^{2}$, where $e$ is the electronic charge, $m_{e 0}$ is the rest mass of the electron and $c$ is the velocity of light in free space. $x_{1}$ occurring in the expression for $B$ in (13) is the real part, supposedly positive, of $\delta_{1}$, corresponding to a growing wave. Furthermore, in (13), while $N$ represents the interaction length $l$ of the gyro-TWT, $\mathrm{I}_{0}$, in the expression for $C$, represents the beam current and the parameter $K$, which is analogous to the interaction impedance of a conventional TWT, is given by

$$
\begin{aligned}
& K= \\
& \frac{\left(\mu_{0} / \varepsilon_{0}\right)^{1 / 2}\left(v_{t} / c\right)^{2}\left(\gamma_{0}^{\mathrm{I}} r_{W}\right)^{2}\left(1+\alpha_{0}^{2}\right) J_{s}^{2}\left\{\gamma_{0}^{\mathrm{I}} r_{H}\right\} J_{s}^{\prime 2}\left\{\gamma_{0}^{\mathrm{I}} r_{L}\right\}}{\pi J_{0}^{2}\left\{\gamma_{0}^{\mathrm{I}} r_{W}\right\}\left(v_{z} / c\right)\left(\beta_{0}^{\mathrm{I}} r_{W}\right)^{4}}
\end{aligned}
$$

where $r_{H}$ and $r_{L}$ are the hollow-beam radius and the Larmor radius, respectively [2], [4], [5].

\section{RESUlTS AND DiscussiON}

The dispersion relation (11) of the disc-loaded cylindrical waveguide, obtained by the present analysis (Section II), has been used in this section to study the effect of the disc parameters on the dispersion characteristics of the disc-loaded 


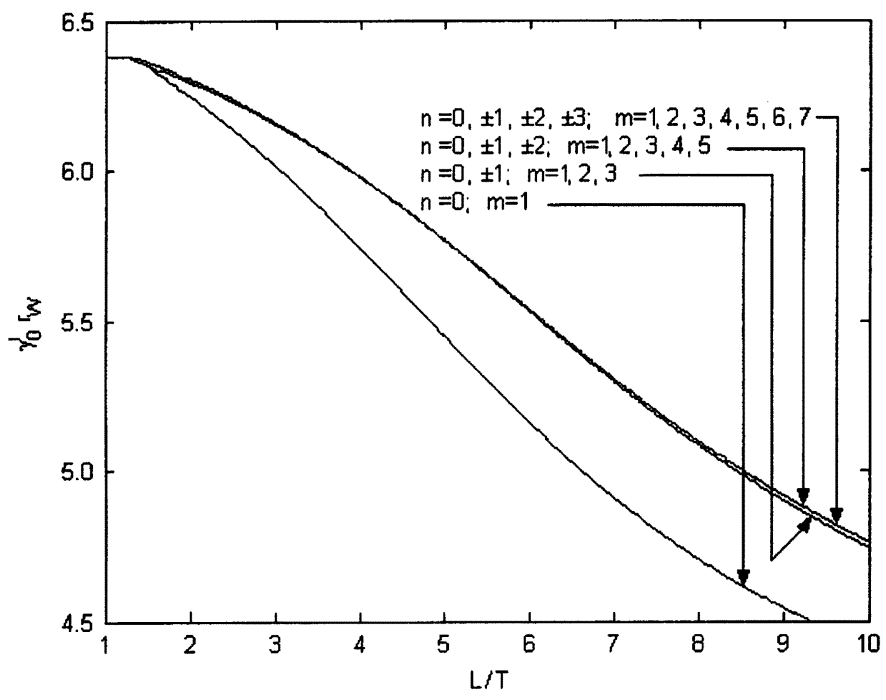

Fig. 2. Convergence of the eigenvalue $\left(\gamma_{0}^{\mathrm{I}} r_{W}\right)$ at $n=0, \pm 1, \pm 2, \pm 3, m=$ $1,2,3,4,5,6,7$ for a wide range of the values of the disc periodicityto-thickness ratio $L / T$, taking typically the disc hole radius relative to the waveguide radius $r_{D} / r_{W}=0.6$, for the mode $\mathrm{TE}_{01}$.

structure, as well as on the gain-frequency response of the disc-loaded gyro-TWT. The dispersion relation (11) that is valid for discs of finite dimension includes all the space harmonics $(n=0, \pm 1, \pm 2, \ldots, \pm \infty)$ in region $\mathrm{I}$, and all the standing-wave modal harmonics $(m=1,2,3, \ldots,+\infty)$ in region II. The dispersion relation is solved using numerical methods in the software MATLAB. The results, with respect to the eigenvalue $\left(\gamma_{0}^{\mathrm{I}} r_{W}\right)$ that would give the cutoff frequency near which a gyro-TWT is operated [5], studied for progressively higher order harmonics, are found to converge at $n=0, \pm 1, \pm 2, \pm 3, m=1,2,3,4,5,6,7$, for a wide range of the values of $L / T$, typically for the $\mathrm{TE}_{01}$ mode (Fig. 2). This suggests that, for the numerical appreciation of the analysis, it would be reasonable enough to truncate the infinite-order determinant of the dispersion relation (11) at $7 \times 7$. Hence, one may study, with the help of (11), the effect of the disc parameters on the shape of the dispersion characteristics that has relevance to attaining wide-band coalescence between the beam-mode and waveguide-mode dispersion characteristics and, consequently, to wide-band gain-frequency response of the gyro-TWT, the latter obtainable from the simple small-signal gain equation (12) through (13) and (14). We have ignored the launching loss in calculation, that is, the first term of (12), as it would not have an appreciable influence on predicting the optimum parameters for wide-band gain-frequency response of the device.

For the special case $r_{D}=r_{W}$, one would have $Z_{0}\left\{\gamma_{m}^{\mathrm{II}} r_{D}\right\}=$ $Z_{0}\left\{\gamma_{m}^{\mathrm{II}} r_{W}\right\} \neq 0$ and $Z_{0}^{\prime}\left\{\gamma_{m}^{\mathrm{II}} r_{D}\right\}=Z_{0}^{\prime}\left\{\gamma_{m}^{\mathrm{II}} r_{W}\right\}=0$, as can be seen from (5). This, since $M_{n m}$ is independent of $r_{D}$ or $r_{W}$, would make the first term of the element of (11) equal to zero, making one read (11) as det $\left|Z_{0}\left\{\gamma_{m}^{\mathrm{II}} r_{W}\right\} J_{0}^{\prime}\left\{\gamma_{n}^{\mathrm{I}} r_{W}\right\}\right|=0$. This, since for this special case one has $Z_{0}\left\{\gamma_{m}^{\mathrm{II}} r_{W}\right\} \neq 0$ as mentioned above, would essentially lead to $J_{0}^{\prime}\left\{\gamma_{n}^{\mathrm{I}} r_{W}\right\}=0$, which may be identified as the dispersion relation of a circular waveguide excited in the $\mathrm{TE}_{0 \mathrm{n}}$ mode of wall radius $=r_{W}$. Similarly, for the special case $L=T$, as can again be seen from (5), one would have $Z_{0}\left\{\gamma_{m}^{\mathrm{II}} r_{D}\right\} \neq 0$ and $Z_{0}^{\prime}\left\{\gamma_{m}^{\mathrm{II}} r_{D}\right\}=0$, and since the function $M_{n m}$ takes on finite values for this special case, following a similar reasoning, one now obtains $J_{0}^{\prime}\left\{\gamma_{n}^{\mathrm{I}} r_{D}\right\}=$ 0 , which may be now identified as the dispersion relation of a circular waveguide excited in the $\mathrm{TE}_{0 \mathrm{n}}$ mode of wall radius $=r_{D}$.

Furthermore, as a special case of $T \rightarrow 0$, that is, for infinitesimally thin discs, the dispersion relation (11) that includes the effect of the finite disc thickness passes on to that obtained earlier by ignoring disc thickness [6]. Also, if the effects of higher order modes were ignored and if one had considered only $n=0$ and $m=1$, over and above the condition $T \rightarrow 0$, the dispersion relation (11) would become $\gamma_{1}^{\mathrm{II}} Z_{0}\left\{\gamma_{1}^{\mathrm{II}} r_{D}\right\} / Z_{0}^{\prime}\left\{\gamma_{1}^{\mathrm{II}} r_{D}\right\}-$ $\gamma_{0}^{\mathrm{I}} J_{0}\left\{\gamma_{0}^{\mathrm{I}} r_{D}\right\} / J_{0}^{\prime}\left\{\gamma_{0}^{\mathrm{I}} r_{D}\right\}=0$, which is identical with that obtained by Choe and Uhm [9].

The dispersion characteristics of a disc-loaded circular waveguide, typically for the $\mathrm{TE}_{01}$ and $\mathrm{TE}_{02}$ modes, obtained by the present analysis, that is, by the dispersion relation (11), very closely agree with those obtained by another theoretical approach based on the coupled-integral-equation technique due to Amari et al. [12], [Fig. 3(a)]. Also, the dispersion characteristics, generated by (11), typically for the $\mathrm{TE}_{01}$ mode, have been validated against the commercial simulation code HFSS, within $0.5 \%$ over the entire passband of the dispersion characteristics, for typical disc parameters [Fig. 3(b)].

The dispersion relation (11) for a disc-loaded circular waveguide obtained by the present analysis, considering the effects of the finite disc thickness and higher order harmonics, has been used here to study the effect of the disc parameters, namely, disc hole radius, disc periodicity and disc thickness on the eigenvalue $\left(\gamma_{0}^{\mathrm{I}} r_{W}\right)$ (Fig. 4) and on the normalized $\omega-\beta$ dispersion characteristics of the structure [Figs. 5(a), 6(a), 7(a)]. A feedback of this study on the dispersion relation (11) has also been interpreted for the gain-frequency response of the disc-loaded gyro-TWT with the help of (12), [Figs. 5(b), 6(b), 7(b)].

The eigenvalue of the disc-loaded circular waveguide remains constant with the disc hole radius up to a value of the latter, beyond which the eigenvalue decreases tending to the value corresponding to the cutoff frequency of the smooth-wall circular waveguide, for all values of the disc periodicity [Fig. 4(a)] and disc thickness [Fig. 4(b)]. Also, for a given disc hole radius, the eigenvalue decreases [Fig. 4(a)] with the increase of the disc periodicity, whereas it increases [Fig. 4(b)] with the increase of the disc thickness. Further, the range of the disc hole radius for the constancy of the eigenvalue increases with the decrease of the disc periodicity [Fig. 4(a)]. However, such range is independent of the disc thickness [Fig. 4(b)].

The effects of the disc hole radius on the dispersion characteristics of the structure considering the effects of the disc thickness $\left(T / r_{W} \neq 0\right)$ and ignoring them $\left(T / r_{W} \rightarrow 0\right)$ are apparent [Fig. 5(a)]. The dispersion characteristics for the special case of the parameter $r_{D} / r_{W}=1.0$ pass on to those for a smooth-wall circular waveguide, and for the special case of the parameter $T / r_{W} \rightarrow 0$, to those reported for infinitesimally thin discs [6], [Fig. 5(a)].

With the decrease of either of the parameters, namely the disc hole radius and the disc periodicity, the lower and upper edge frequencies of the passband of the dispersion characteristics both increase though to unequal extents such that the passband decreases or increases according as the disc hole radius 


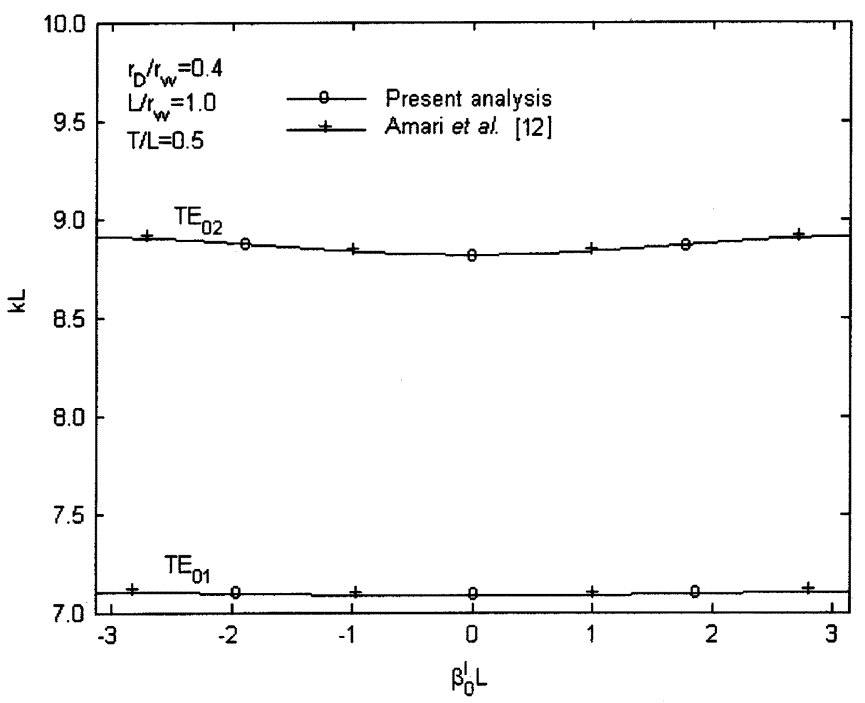

(a)

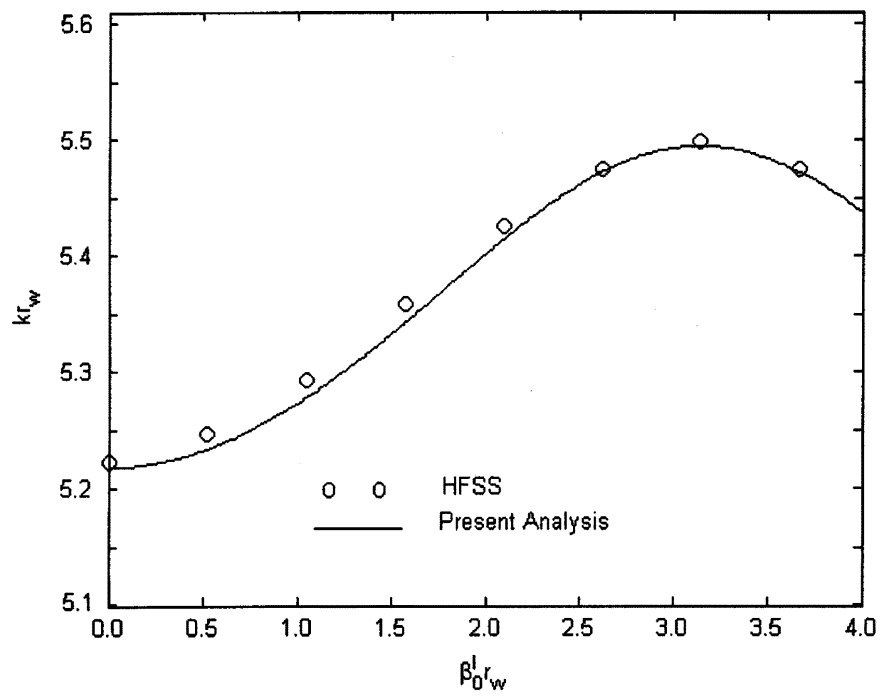

(b)

Fig. 3. Validation of the dispersion characteristics of a disc-loaded circular waveguide obtained by the present analysis against those obtained (a) by the coupled-integral-equation technique due to Amari et al. [12], for typical disc parameters $r_{D} / r_{W}=0.4, L / r_{W}=1.0$, and $T / L=0.5$, for the $\mathrm{TE}_{01}$ and $\mathrm{TE}_{02}$ modes and (b) by HFSS, for typical disc parameters $r_{D} / r_{W}=$ $0.6, L / r_{W}=1.0$, and $T / L=0.3$, for the $\mathrm{TE}_{01}$ mode.

or the disc periodicity decreases, the midband frequency of the passband, however, shifting to a higher value for the decrease of both the parameters [Figs. 5(a), 6(a)]. It is noted that, although both the upper and lower edge frequencies of the passband depend on the disc hole radius for discs of finite thickness, it is the lower edge, and not the upper edge frequency, of the passband that depends on the disc hole radius for infinitesimally thin discs [Fig. 5(a)]. Furthermore, there exists an optimum value of the disc hole radius corresponding to the maximum frequency range of the straight line portion of the dispersion characteristics. No such optimum value however exists for the disc periodicity. With the decrease of the latter, the frequency range of the straight line portion of the dispersion characteristics increases and shifts away from the lower cutoff frequency [Fig. 5(a)].

Similarly, with the decrease of the disc thickness, the lower and upper edge frequencies of the passband in the dispersion

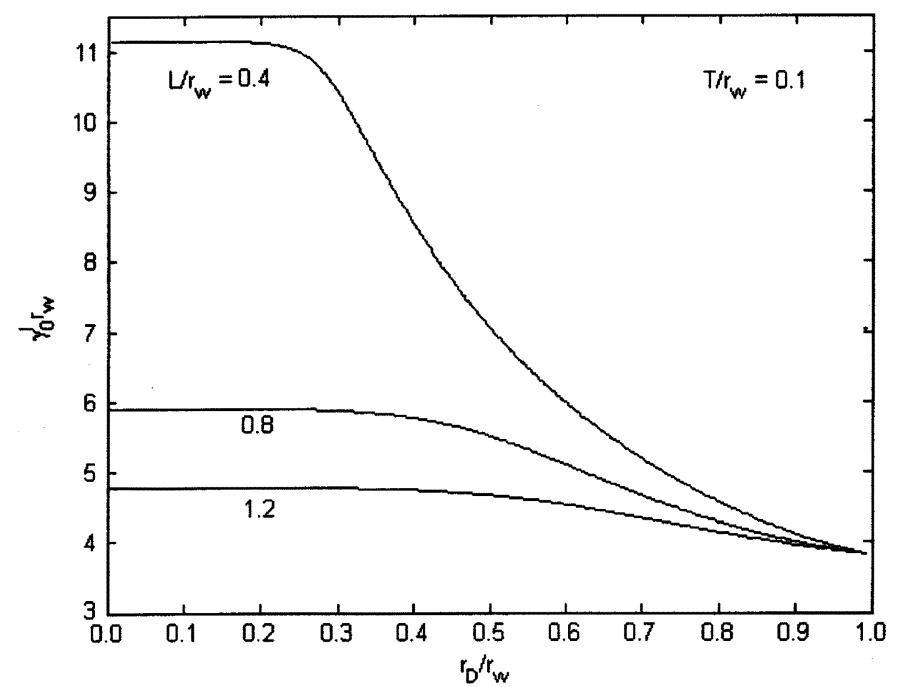

(a)

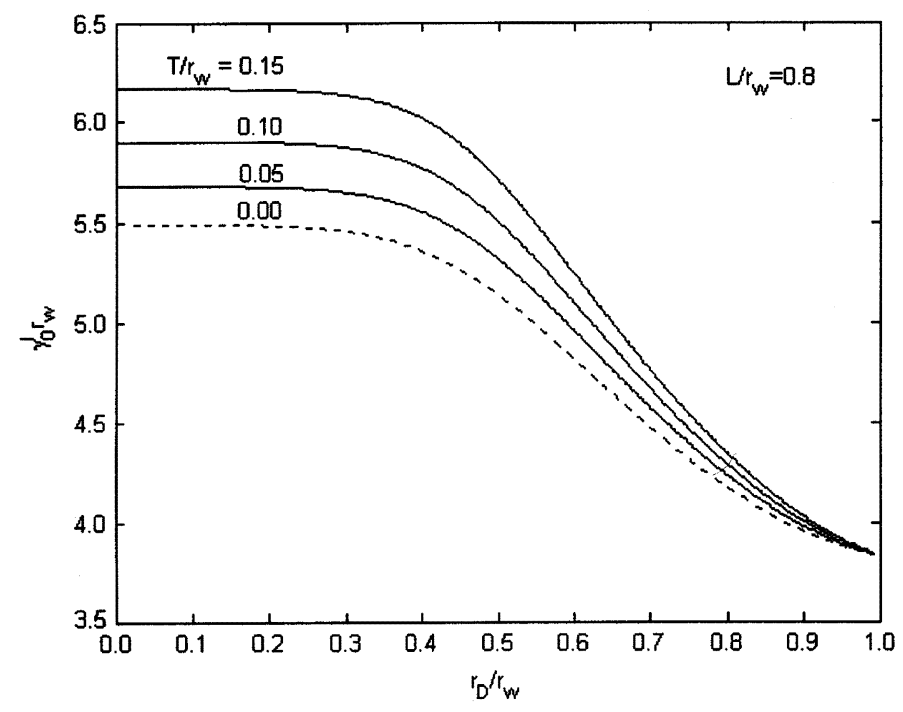

(b)

Fig. 4. Eigenvalue-versus-disc hole radius taking (a) disc periodicity and (b) disc thickness as parameters, typically for the $\mathrm{TE}_{01}$ mode. The broken curve (b) refers to infinitesimally thin discs [6].

characteristics both decrease such that the passband first decreases and then increases passing through a minimum; and the midband frequency of the passband, as well as the frequency corresponding to the beginning of the straight line portion of the dispersion characteristics, shifts to a lower value [Fig. 7(a)]. As the disc thickness is increased, the eigenvalue increases [Fig. 4(b)] and, consequently, the frequency corresponding to the maximum device gain shifts to a higher value [Fig. 7(b)]. The dispersion characteristics taking the disc thickness as the parameter have been grouped for relatively thin and relatively thick discs [Fig. 7(a)]. The dispersion characteristics for the special case of infinitesimally thin discs $\left(T / r_{W} \rightarrow 0\right)$ pass on to those reported ignoring the effect of finite disc thickness [6]. Similarly, the dispersion characteristics for the special case of disc thickness tending to disc periodicity $\left(L / r_{W} \rightarrow T / r_{W}\right.$ or $T / L \rightarrow 1$ ) passes on to those for a smooth-wall circular waveguide of wall radius equal to the disc hole radius [Fig. 7(a)]. The shape of the dispersion characteristics depends on the disc 


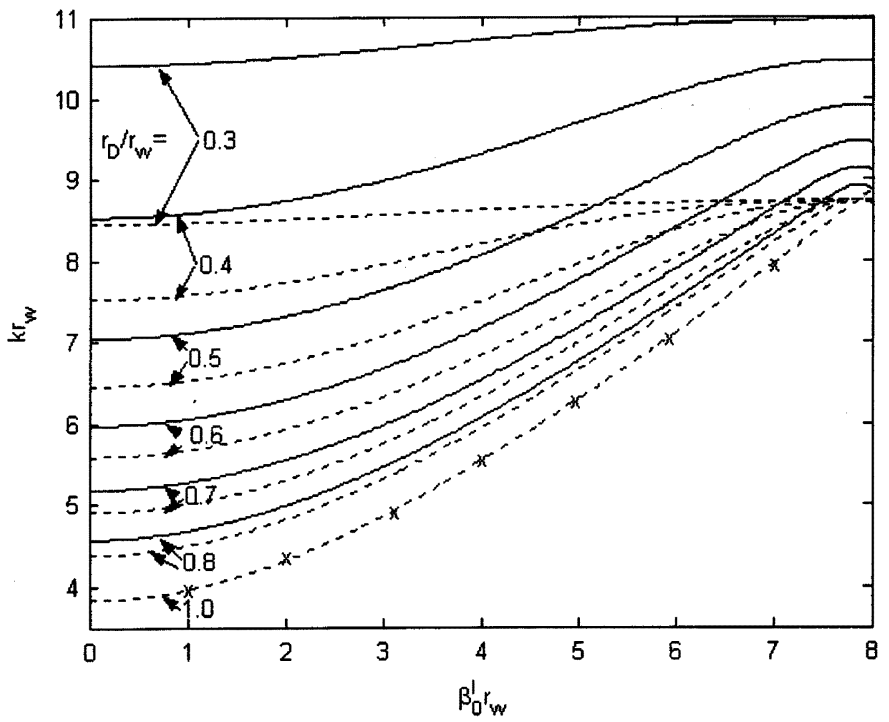

(a)

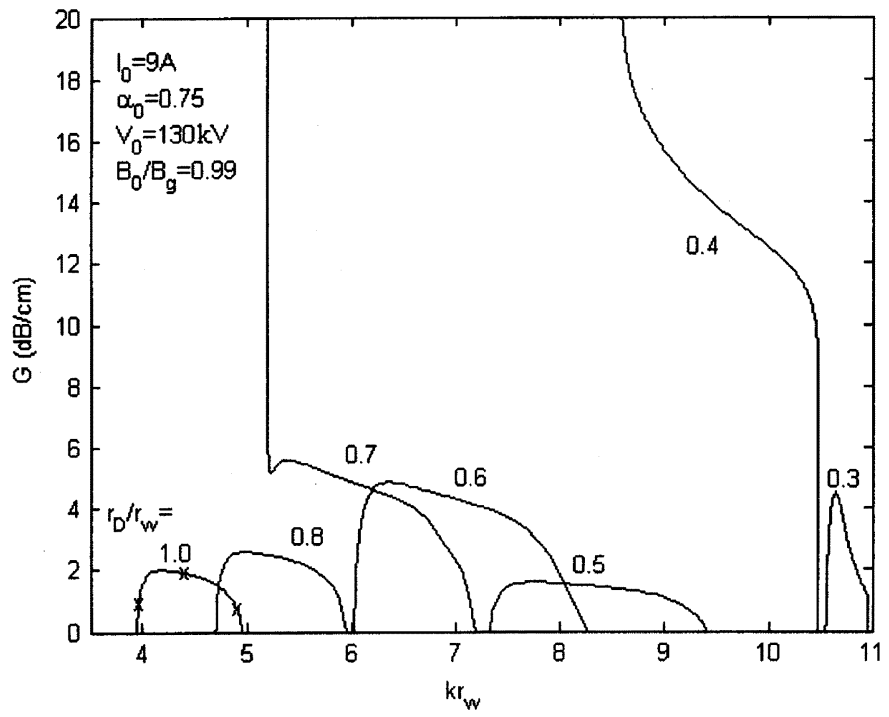

(b)

Fig. 5. (a) Dispersion characteristics of the disc-loaded circular waveguide typically for the $\mathrm{TE}_{01}$ mode, taking disc hole radius as the parameter for thick $\left(T / r_{W}=0.1\right)$ (solid line) and infinitesimally thin $\left(T / r_{W}=0\right)$ (broken line) discs [6], for a typical disc periodicity $L / r_{W}=0.4$, and (b) the corresponding gain-frequency response for thick $\left(T / r_{W}=0.1\right)$ discs. For the parameter $r_{D} / r_{W}=1.0$, the broken line with crosses in dispersion characteristics (a) and the solid line with crosses in gain-frequency response (b) refer to a smooth-wall circular waveguide. Beam and magnetic field parameters are: $\mathrm{I}_{0}=9.0 \mathrm{~A}$, $\mathrm{V}_{0}=130 \mathrm{kV}, \alpha_{0}=0.75, r_{H}=0.48 r_{D}$, and $B_{0} / B_{g}=0.99$.

thickness but not as much as it does on the disc hole radius or disc periodicity [Figs. 5(a), 6(a), 7(a)].

Since the axial phase propagation constant $\left(\beta_{0}^{\mathrm{I}}\right)$ obtained from the cold (beam-absent) analysis of the disc-loaded waveguide has been fed back in the small-signal gain equation (12) of the gyro-TWT, it is expected that the behavior of the dispersion characteristics of the structure [Figs. 5(a), 6(a), 7(a)] would be reflected in the gain-frequency response of the device [Figs. 5(b), 6(b), 7(b)]. For instance, the existence of the optimum value of the disc hole radius for the maximum frequency range of the straight line portion of the dispersion characteristics, which in turn would correspond to the maximum bandwidth of coalescence between the beam-mode and waveguide-mode dispersion

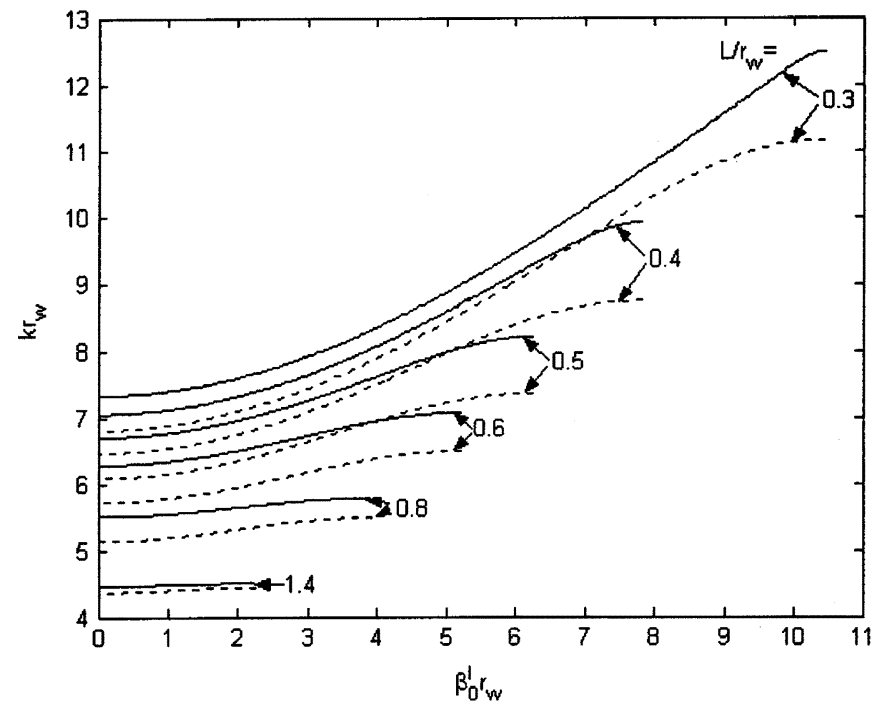

(a)

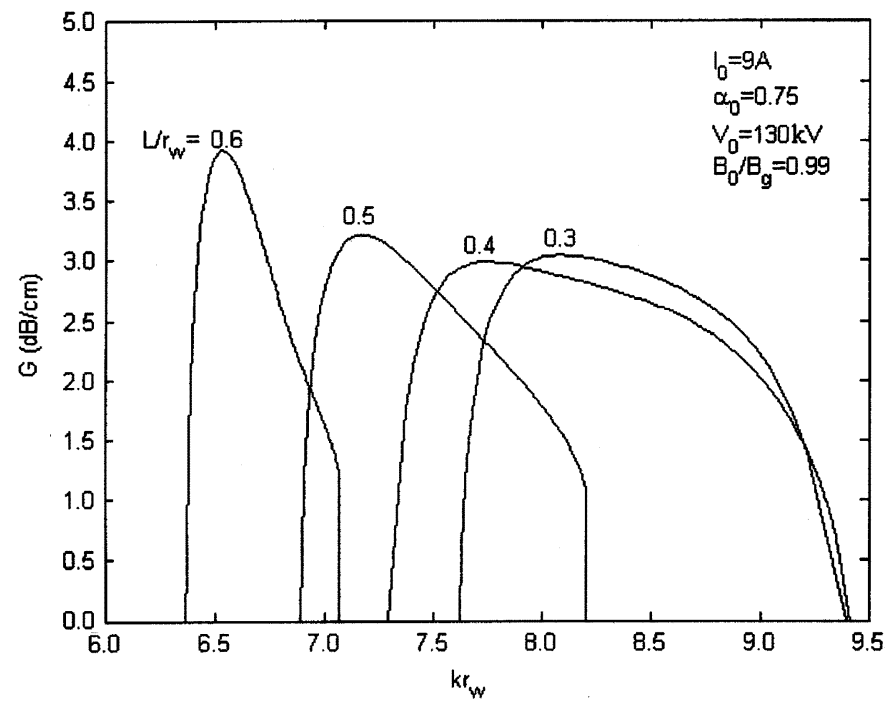

(b)

Fig. 6. (a) Dispersion characteristics of the disc-loaded circular waveguide typically for the $\mathrm{TE}_{01}$ mode, taking disc periodicity as the parameter for thick $\left(T / r_{W}=0.1\right)$ (solid line) and infinitesimally thin $\left(T / r_{W}=0\right)$ (broken line) discs [6], for a typical disc hole radius $r_{D} / r_{W}=0.5$, and (b) the corresponding gain-frequency response for thick $\left(T / r_{W}=0.1\right)$ discs. Beam and magnetic field parameters are as in Fig. 5.

characteristics of a gyro-TWT, or the increase of such frequency range with the decrease of the disc periodicity [Figs. 5(a), 6(a)] is immediately reflected in the bandwidth of the gyro-TWT [Figs. 5(b), 6(b)]. Thus, one obtains an optimum value of the disc hole radius corresponding to the maximum device bandwidth [Fig. 5(b)] and an increase in the device bandwidth, though at the cost of the device gain, with a decrease of the disc periodicity [Fig. 6(b)]. An optimum value of the disc hole radius is also obtained for the maximum device gain. The midband frequency of amplification is found to be sensitive to the value of the disc hole radius, increasing with the decrease of the latter [Fig. 5(b)]. [The disc hole radius that makes the gain shoot up to a very high value [Fig. 5(b)] corresponds to the operating frequency approaching the cutoff frequency where the phase propagation constant $\beta_{0}^{\mathrm{I}} \rightarrow 0$ [Fig. 5(a)], which makes $K$ and hence $G$ very high, as can be appreciated by examining (12) through (14)]. 


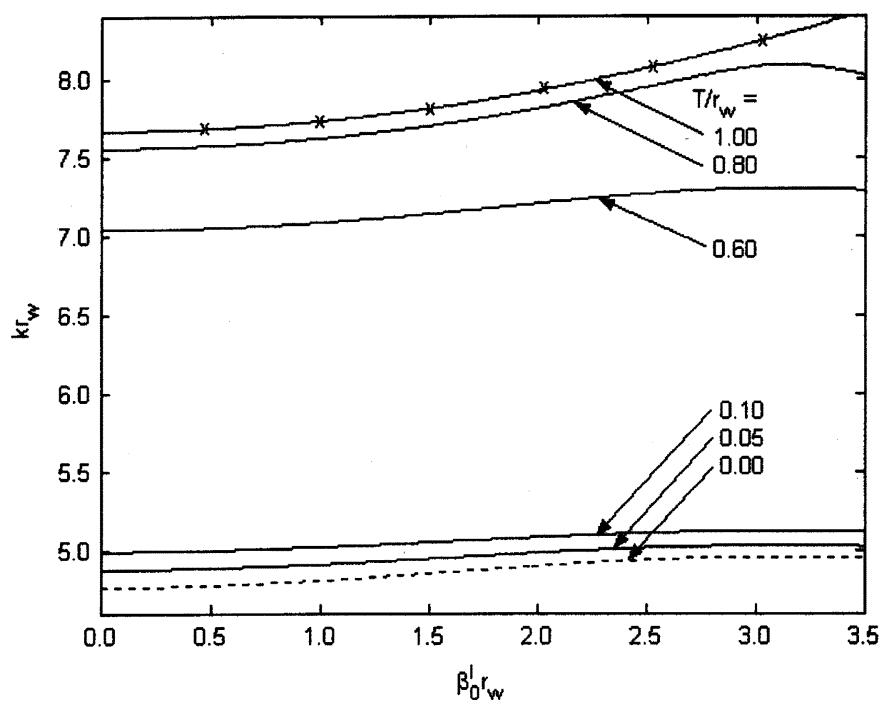

(a)

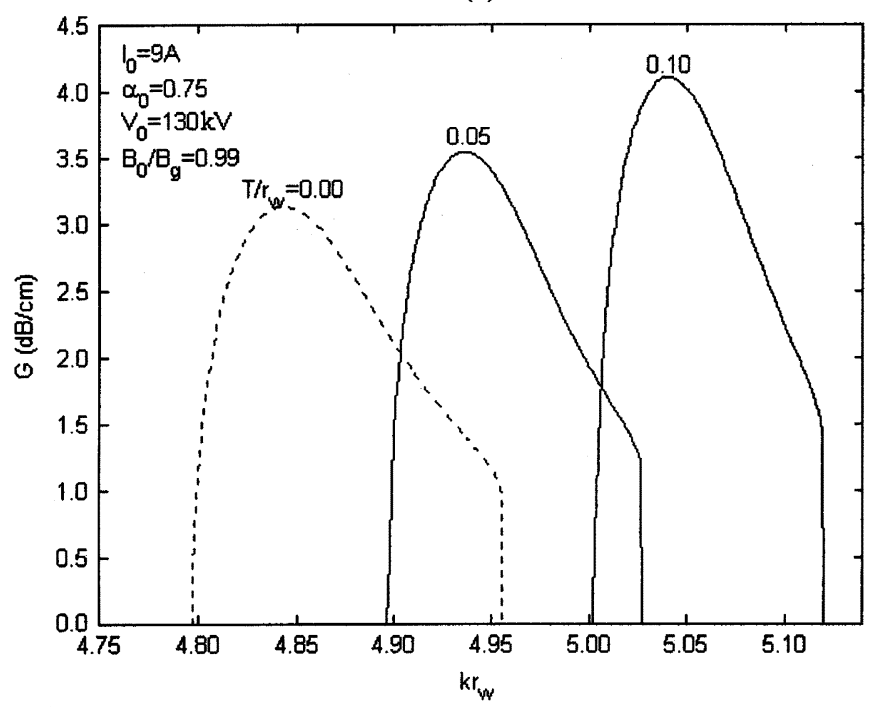

(b)

Fig. 7. (a) Dispersion characteristics of the disc-loaded circular waveguide typically for the $\mathrm{TE}_{01}$ mode, taking disc thickness as the parameter for thick (solid line) and infinitesimally thin $\left(T / r_{W}=0\right)$ (broken line) discs [6], for typical disc parameters $L / r_{W}=1.0$ and $r_{D} / r_{W}=0.5$, and (b) the corresponding gain-frequency response for thick discs. Dispersion characteristics for the case of disc thickness equal to disc periodicity $\left(L / r_{W}=T / r_{W}=1.0\right)$ (solid line with crosses) refer to a smooth-wall circular waveguide of wall radius $r_{W}=r_{D}$. Beam and magnetic field parameters are as in Fig. 5.

Since the shape of the dispersion characteristics is relatively less dependant on the disc thickness [Fig. 7(a)], the device bandwidth depends less on the disc thickness than on the other disc parameters, namely, the disc hole radius and the disc periodicity. With the increase of the disc thickness, though the device bandwidth has slight tendency to decrease, the device gain increases [Fig. 7(b)]. Thus, one should go for thin and well separated discs for larger device gain, and thick and close by discs for wider device bandwidths [Figs. 6(b), 7(b)]. Furthermore, out of the three parameters, namely, the disc hole radius, disc periodicity, and disc thickness, the disc periodicity is the most effective parameter in controlling the device bandwidth whereas the disc hole radius is the most effective parameter in controlling the device gain of a disc-loaded gyro-TWT. Also, the maximum

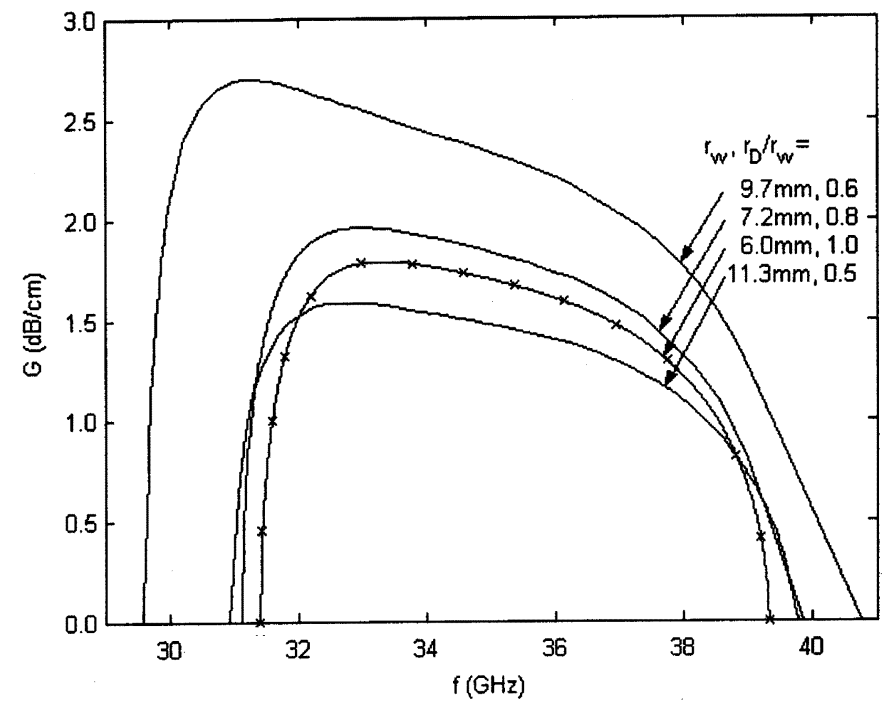

Fig. 8. Optimization of the disc parameters for the maximum device gain or device bandwidth without causing a change in the midband frequency, for instance, around $35 \mathrm{GHz}$ in the atmospheric window. Beam and magnetic field parameters are as in Fig. 5.

device bandwidth is achieved by controlling the disc parameters though at the cost of the device gain, and vice versa.

The control of the disc parameters over the device gain or bandwidth is in general accompanied by a change in the center frequency of the amplification band. This may call for optimizing the disc parameters for the maximum device gain or bandwidth without causing any change in the midband frequency of the amplification band; for instance, typically around $35 \mathrm{GHz}$ (atmospheric window) (Fig. 8). Thus, choosing to keep the disc thickness and disc periodicity unchanged, one may optimize the waveguide radius and disc hole radius for the purpose of either widening the device bandwidth or enhancing the device gain, the optimum parameters (waveguide radius and disc hole radius) obviously coming out to be different for the two purposes (Fig. 8). For either of these purposes, the wall radius of a disc-loaded circular waveguide would be greater than that of a smooth-wall circular waveguide (Fig. 8).

\section{CONCLUSION}

The analysis of a disc-loaded circular waveguide developed in this paper is carried out in the fast-wave regime and thus is helpful in designing wide-band gyro-TWTs. The analysis of the structure is generalized considering 1) higher order modal harmonics in the disc-occupied region, supporting standing waves, and higher order space harmonics, arising from the axial periodicity of discs, in the disc-free region, and 2) the effects of finite disc thickness. The optimum disc parameters (hole radius, periodicity, and thickness) predicted for maximum coalescence bandwidth between the beam-mode and waveguide-mode dispersion characteristics, corresponding to the widest frequency band over which the dispersion curve of the structure could be straightened, agree with the optimum disc parameters predicted for maximum device bandwidth.

The optimization of the disc periodicity is found to be more effective than that of the other disc parameters, namely, the disc hole radius and disc thickness in maximizing the device bandwidth. On the other hand, the disc hole radius proves to be the 
most effective optimizing parameter in maximizing the device gain. However, care needs to be taken in selecting the disc parameters for the desired gain and bandwidth, since any change in the disc parameters is, in general, accompanied by a change in the center frequency of the pass band of the structure and, hence, also in the center frequency of amplification band of the device.

It is believed that, although the large-signal analysis would have given a more accurate estimate of the gain of the device, the results of the present cold analysis of the disc-loaded circular waveguide considering the effect of the finite disc thickness and the feedback thereof into the small-signal gain-frequency response could be helpful in optimizing the design of a gyro-TWT for its wide-band performance.

\section{REFERENCES}

[1] A. V. Gaponov-Grekhov and V. L. Granatstein, Applications of HighPower Microwaves. London, U.K.: Artech House, 1994.

[2] B. N. Basu, Electromagnetic Theory and Applications in Beam-Wave Electronics, Singapore: World Scientific, 1996.

[3] K. R. Chu, Y. Y. Lau, L. R. Barnett, and V. L. Granatstein, "Theory of a wide-band distributed gyrotron traveling wave amplifier," IEEE Trans. Electron Devices, vol. 28, no. 4, pp. 866-871, Apr. 1981.

[4] M. Agrawal, G. Singh, P. K. Jain, and B. N. Basu, "Analysis of a tapered vane loaded broad-band gyro-TWT," IEEE Trans. Plasma Sci., vol. 29, no. 3, pp. 439-444, Jun. 2001.

[5] S. J. Rao, P. K. Jain, and B. N. Basu, "Broadbanding of a gyro-TWT by dielectric-loading through dispersion shaping," IEEE Trans. Electron Devices, vol. 43, no. 12, pp. 2290-2299, Dec. 1996.

[6] V. Kesari, P. K. Jain, and B. N. Basu, "Approaches to the analysis of a disc-loaded cylindrical waveguide for potential application in wide-band gyro-TWTs," IEEE Trans. Plasma Sci., vol. 32, no. 5, pp. 2144-2151, Oct. 2004.

[7] E. L. Chu and W. W. Hansen, "The theory of disc-loaded waveguides," J. Appl. Phys., vol. 18, pp. 996-1008, Nov. 1947.

[8] D. A. Watkins, Topics in Electromagnetic Theory. New York: Wiley, 1958.

[9] J. Y. Choe and H. S. Uhm, "Theory of gyrotron amplifiers in disc or helix-loaded waveguides," Int. J. Electron., vol. 53, no. 6, pp. 729-741, Sep. 1982.

[10] P. J. B. Clarricoats and A. D. Olver, Corrugated Horns for Microwave Antennas. London, U.K.: Peter Peregrinus, 1984.

[11] R. B. Dybdal Jr., L. Peters, and W. H. Peake, "Rectangular waveguides with impedance walls," IEEE Trans. Microw. Theory Tech., vol. MTT-39, no. 1, pp. 2-8, Jan. 1971.

[12] S. Amari, R. Vahldieck, and J. Bornemann, "Analysis of propagation in periodically loaded circular waveguides," in IEE Proc. Microw. Antennas Propag., vol. 146, Feb. 1999.

[13] S. Amari, R. Vahldieck, J. Bornemann, and P. Leuchtmann, "Spectrum of corrugated and periodically loaded waveguides from classical matrix eigenvalues," IEEE Trans. Microw. Theory Tech., vol. 48, no. 3, pp. 453-460, Mar. 2000.

[14] J. Esteban and J. M. Rebollar, "Characterization of corrugated waveguides by modal analysis," IEEE Trans. Microw. Theory Tech., vol. 39, no. 6, pp. 937-943, Jun. 1991.

[15] D. Wagner, M. Thumm, and W. Kasparek, "Hybrid modes in highly oversized corrugated rectangular waveguides," Int. J. Infrared Millimeter Waves, vol. 20, no. 4, pp. 567-581, Apr. 1999.

[16] A. W. Fliflet, "Linear and nonlinear theory of the Doppler-shifted cyclotron resonance maser based on TE and TM waveguide modes," Int. J. Electron., vol. 61, no. 6, pp. 1049-1080, Sep. 1986.

[17] J. R. Pierce, Traveling-Wave Tubes. New York: D. Van Nostrand, 1950.

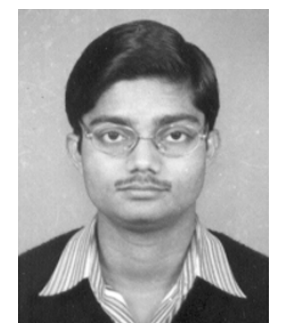

Vishal Kesari was born in Mughalsarai (Uttar Pradesh), India, in 1978. He received M.Sc. degree in physics from Purvanchal University, Jaunpur, India, in 2001 . He is currently working toward the Ph.D. degree in the Department of Electronics Engineering, the Institute of Technology, Banaras Hindu University, Varanasi, India, in the area of microwave tubes, as Junior Research Fellow under CAS (UGC) program.

His research interest includes periodically loaded interaction structures for slow- and fast-wave vacuum electron devices.

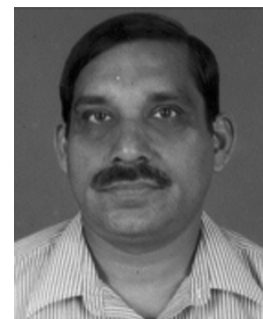

P. K. Jain received the B.Tech. degree in electronics engineering, and M.Tech. and Ph.D. degrees in microwave engineering, all from Banaras Hindu University (BHU), Varanasi, India, in 1979, 1981, and 1988, respectively.

In 1981, he joined Centre of Research in Microwave Tubes, Department of Electronics Engineering, Institute of Technology, BHU, as Lecturer, and is currently working there as Professor. $\mathrm{He}$ was Principal Investigator of the project, "Studies on slow- and fast-wave interaction structures for beam-wave interaction in TWTs," sponsored by Ministry of Defence. The areas of his current research and publication include CAD/CAM, modeling and simulation of microwave tubes and their subassemblies, including broadbanding of helix traveling-wave tubes (TWTs), cyclotron resonance measure devices, including gyrotrons, and gyro-TWTs and their performance improvement.

Dr. Jain is Fellow of the Institution of Electronics and Telecommunication Engineers (India).

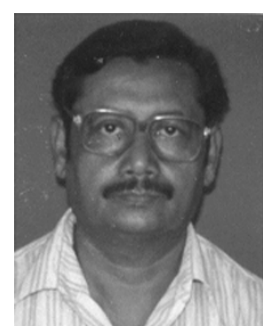

B. N. Basu received the M.Tech. and Ph.D. degrees from the Institute of Radiophysics and Electronics, Calcutta University, India, in 1966 and 1976, respectively.

$\mathrm{He}$ is a Professor at the Centre of Research in Microwave Tubes, Electronics Engineering Department, Banaras Hindu University, Varanasi, India. He was associated with Central Electronics Engineering Research Institute (CEERI), Pilani, Council of Scientific and Industrial Research (CSIR), India as Distinguished Visiting Scientist of CSIR. He was the partner with University of Lancaster (UoL), U.K. and CEERI under Academic Link and Interchange Scheme (ALIS) between CSIR and British Council and worked at UoL. Also, he was with Seoul National University, Seoul, Korea, as Visiting Scientist. His areas of current research and publications include helix-TWTs, gyrotrons, and gyro-TWTs. He has authored or coauthored around 100 research papers in peer-reviewed journals and authored a book entitled Electromagnetic Theory and Applications in Beam-Wave Electronics (World Scientific, Singapore, 1996).

Dr. Basu is Member of Technical Committee on Vacuum Electron Devices of IEEE Electron Devices Society. He is Fellow and recipient of S.V.C. Aiya Memorial award for outstanding contributions in motivating research, of Institution of Electronics and Telecommunication Engineers, India. 